\title{
MODELO ANALÍTICO PARA DETERMINAR LA INFILTRACIÓN CON BASE EN LA LLUVIA MENSUAL
}

\author{
Gunther Schosinsky \& Marcelino Losilla \\ Escuela Centroamericana de Geología, Apdo. 2-14, 2060 Universidad de Costa Rica, San José
}

(Recibido 12/1/1999; Aceptado 13/8/1999)

\begin{abstract}
Several thesis studies have been completed in Costa Rica, that evaluate the recharge to aquifers using pluviographic rainfall records and infiltration tests of soils. In this study, an equation has been developed to describe recharge using base infiltration and monthly precipitation data. The rainfall was divided into runoff and infiltration components. The rainfall data (measured every 10 minutes) that were in excess of base infiltration were considered as surface runoff and the remainder was considered as potential infiltration. The summation of these calculated recharge values produced a monthly recharge that potentially could infiltrate into the soil. The pluviographic records analyzed in the thesis works, were obtained from the following rainfall stations: Aeropuerto Juan Santamaría, Puntarenas and Palmira. A similar work was done in SENARA (National Service of Ground Water) using the rainfall station of Hacienda Tempisque.

From the thesis works and existing literature of runoff coefficients data, correlation was done in order to develop an equation to evaluate the infiltration, which took into account rainfall interception on vegetation, base infiltration of soils, vegetative cover and surface topography.

In this way, a potential infiltration equation is obtained (the calculation of infiltration based on rainfall intensity and soil texture) for the mentioned zones. The infiltration coefficient was determinated by correlating data from, achieving a correlation coefficient $\left(\mathrm{R}^{2}\right)$ of 0.99 .

A general infiltration equation for Costa Rica was obtained, in which the effects of topography, surface vegetation and rainfall retention were included. The values of the potential infiltration obtained with the general equation was compared with the infiltration values obtained from the rainfall records. The equation has an error of $13 \%$ with $68 \%$ of confiability and an error of $26 \%$ with $95 \%$ of confiability.

This method simplifies the procedure to estimate infiltration, which is needed to determine the recharge of aquifers using the base infiltration of the soils and the mean monthly rainfall of the zone. Without the equation, the recharge should be estimated with the daily analysis of the rainfall records. Possibly this equation could be used in Central America with similar climate.
\end{abstract}

RESUMEN: Para estimar la recarga de los acuíferos en Costa Rica, se han realizado varios trabajos de tesis utilizando la distribución de precipitación y de infiltración básica de los suelos, donde se analizaron bandas pluviográficas y se obtuvieron las intensidades de lluvia, que fueron comparadas con los diferentes valores de infiltración básica, separando la lluvia en escorrentía superficial e infiltración potencial. Asignándole a la escorrentía el valor del exceso de intensidad de lluvia mayor que el valor de infiltración básica obtenida en el campo. A la infiltración potencial, se asignaron aquellos milímetros de intensidad de lluvia menores que el valor de infiltración básica analizado. De esta forma se obtiene la cantidad de agua que infiltra potencialmente durante el mes, para cada valor de infiltración básica utilizado. Los análisis de bandas que se realizaron en los trabajos de tésis corresponden a las estaciones meteorológicas de Aeropuerto Juan Santamaría, Puntarenas y Palmira. Además se realiza una investigación similar en el SENAS (Servicio Nacional de Aguas Subterráneas) utilizando la estación Hacienda Tempisque.

Además, de la información recopilada de la lectura de bandas, se analizaron los coeficientes de escorrentía, propuestos por otros autores, para así desarrollar una ecuación que permita estimar la infiltración, integrando la interceptación de lluvia en las hojas, la infiltración básica del suelo, cobertura de la superficie y la topografía.

De esta forma se obtiene una ecuación de la infiltración potencial (infiltración debida únicamente a la intensidad de lluvia y a la textura del suelo) para cada uno de estos lugares, mediante un coeficiente de infiltración. Este coeficiente, se determina mediante correlaciones de los datos obtenidos del análisis de bandas. Las correlaciones utilizadas, mostraron coeficientes de correlación $\left(\mathrm{R}^{2}\right)$ de 0,99 .

Posteriormente se obtiene una ecuación general de la infiltración de lluvia en Costa Rica, la que se ajusta por pendiente topográfica, cobertura vegetal y interceptación de lluvia en las hojas. El valor de la infiltración potencial obtenida por la ecuación general, comparado con los valores de infiltración obtenidos por bandas, muestra un error de un $13 \%$ y $26 \%$ para una confiabilidad del $68 \%$ y $95 \%$ respectivamente.

Este método simplifica enormemente el procedimiento para el cálculo de la precipitación que infiltra, la que es necesaria conocer, para determinar la recarga a los acuíferos, utilizando la infiltración básica de los suelos y la precipitación mensual de la zona. El no contar con dicha ecuación, implicaría realizar análisis diarios de las bandas pluviográficas. Esta ecuación, posiblemente puede utilizarse en algunas regiones de Centro América, donde el clima es similar. 


\section{INTRODUCCIÓN}

La necesidad de estimar la recarga de los acuíferos en los proyectos de evaluación de las aguas subterráneas, ha hecho que en Costa Rica se realicen estudios tendientes a establecer dicha recarga con base en la distribución de precipitación y coeficientes de infiltración en los suelos del país.

Para evaluar la infiltración en una zona, se determinan los diferentes valores de capacidad de infiltración básica de los suelos. Posteriormente se analizan bandas de pluviógrafos, determinando las intensidades de lluvia de los aguaceros ocurridos durante un día, que sumados forman los aguaceros de un mes, hasta analizar uno o más años. De las bandas, se leen las intensidades de lluvia, obtenida en períodos de diez minutos, hasta completar la duración de cada uno de los aguaceros ocurridos. Estas intensidades son comparadas con los diferentes valores de infiltración básica, separando la lluvia en escorrentía superficial e infiltración potencial. Asignándole a la escorrentía, los valores de intensidad de lluvia mayores que el valor de infiltración básica obtenida en el campo y a la infiltración potencial, aquellos milímetros de intensidad de lluvia menores que el valor de infiltración básica analizado. De esta forma se obtiene la cantidad de agua que infiltra potencialmente durante el mes, para cada valor de infiltración básica utilizado.

El primer análisis de bandas, con este objetivo, fue realizado por el SENAS (Servicio Nacional de Aguas Subterráneas) para la zona del valle del río Tempisque (Elizondo, 1982), utilizando los datos de la estación meteorológica Hacienda Tempisque. Posteriormente, en trabajos de tesis, siguiendo la misma metodología utilizada en el Tempisque, se llevaron a cabo estudios similares para las zonas de San Antonio de Belén (Gómez, 1987), Barranca (Arredondo, 1995), y Zarcero (Montero \& Solano, 1986) utilizando los datos de las estaciones meteorológicas del Aeropuerto Juan Santamaría, Puntarenas y Palmira, respectivamente.

Dada la cantidad de aguaceros analizados, se propone establecer una ecuación que permita derivar la infiltración de una lluvia mensual, utilizando los datos obtenidos del análisis de las bandas pluviográficas. La metodología utilizada, es la recolección de los datos analizados de las bandas y establecer correlaciones entre la infiltración potencial mensual, el valor de infiltración básica del suelo y la precipitación mensual. Además, los valores de infiltración son ajustados por la pendiente topográfica, cobertura vegetal y interceptación de lluvia en las hojas. Para esto, se analizaron los coeficientes de escorrentía de la literatura existente, para integrarlos dentro de la ecuación desarrollada.

\section{COEFICIENTES DE INFILTRACIÓN}

El "Manual de Instrucciones de Estudios Hidrológicos" realizado por las Naciones Unidas, con colaboración de los gobiernos de El Salvador, Guatemala, Honduras, Nicaragua, Panamá y Costa Rica proponen la siguiente ecuación para el análisis del coeficiente de infiltración aparente, que corresponde a la fracción de lluvia que aparentemente se infiltra:

$$
\begin{array}{ll}
\mathrm{C}=\left(\mathrm{Kp}+\mathrm{Kv}+\mathrm{K}_{\mathrm{fc}}\right) & \\
\mathrm{C} \text { [tanto por uno }] & =\text { Coeficiente de infiltración. } \\
\mathrm{Kp}[\text { tanto por uno] } & \text { Fracción que infiltra por efecto } \\
& \text { de pendiente. } \\
\mathrm{Kv}[\text { tanto por uno] } & \text { Fracción que infiltra por efecto } \\
& \text { de cobertura vegetal. } \\
\mathrm{K}_{\mathrm{fc}}[\text { tanto por uno] } & \text { Fracción que infiltra por tex tura } \\
& \text { del suelo. }
\end{array}
$$

El cuadro 1 muestra los valores que componen el coeficiente de infiltración sugeridos en el Manual de Estudios Hidrológicos (ONU, 1972).

Se analizaron los índices de escorrentía sugeridos por Ven Te Chow (1994), los que son utilizados en la ciudad de Austin (Texas, USA). En estos índices, se considera que el coeficiente de infiltración aparente ( 1 - coeficiente de escorrentía) disminuye aproximadamente en 0,04 al pasar las pendientes del terreno entre 2 y $7 \%$ a superiores de $7 \%$. Los terrenos con una pobre cubierta de zacate (menos del 50\%) tienen un coeficiente de infiltración levemente superior al de 
Cuadro1

Componentes del coeficiente de infiltración (ONU,1974)

\begin{tabular}{|c|c|c|}
\hline \multicolumn{2}{|c|}{ Por textura de suelo } & $\mathrm{K}_{\mathrm{fc}}$ \\
\hline \multicolumn{2}{|c|}{ Arcilla compacta impermeable } & 0,10 \\
\hline \multicolumn{2}{|c|}{ Combinación de limo y arcilla } & 0,20 \\
\hline \multicolumn{2}{|c|}{ Suelo limo arenoso no muy compacto } & 0,40 \\
\hline \multicolumn{2}{|c|}{ Por pendiente } & $\mathrm{Kp}$ \\
\hline Plana & $0.02 \%-0.06 \%$ & 0,30 \\
\hline Moderada & $0.3 \%-0.4 \%$ & 0,20 \\
\hline Colinas & $3 \%-4 \%$ & 0,10 \\
\hline \multicolumn{2}{|c|}{ Por cobertura vegetal } & $\mathrm{Kv}$ \\
\hline \multicolumn{2}{|c|}{ Terrenos cultivados } & 0,10 \\
\hline \multicolumn{2}{|l|}{ Bosques } & 0,20 \\
\hline
\end{tabular}

los terrenos cultivados, aumentando aproximadamente en 0.01 en los terrenos cultivados. Además, los terrenos con cubierta de zacate (más del $75 \%$ ) o cubiertos con bosque, tienen coeficientes de infiltración similares entre sí, disminuyendo aproximadamente en 0,01 en terrenos con bosque. Los terrenos cubiertos con pastizal disminuyen el coeficiente de infiltración aproximadamente en 0,02 con respecto al del bosque. Basado en los incrementos anteriores de Ven Te Chow y considerando los coeficientes de la ONU, se proponen los siguientes coeficientes de infiltración (Cuadro 2):

Cuadro 2

Coeficientes de infiltración propuestos

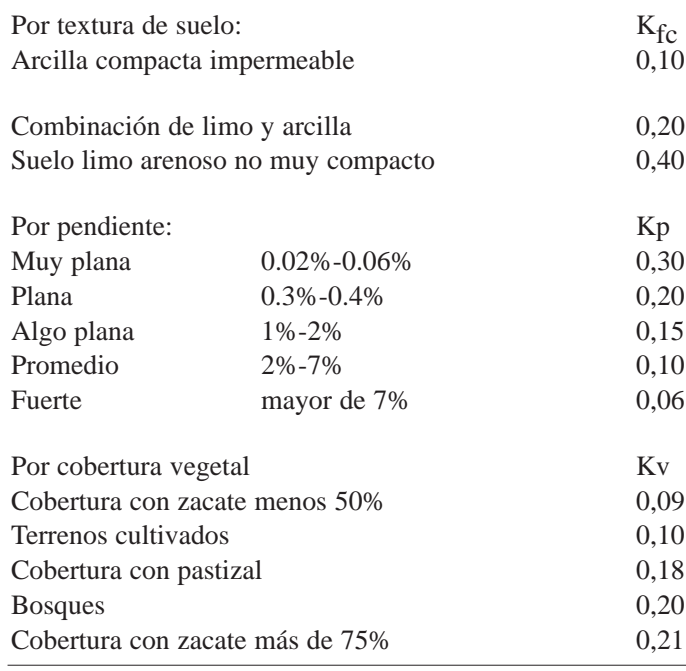

\section{INFILTRACIÓN}

De la lluvia que llega a la superficie del suelo, una fracción de ella infiltra, otra escurre y una pequeña fracción queda en charcos, que termina evaporándose o infiltrando. Sin embargo, la única fracción de lluvia con potencial a infiltrarse es la que llega a la superficie del suelo. Otra fracción de lluvia a considerar; es la interceptada por el follaje de las plantas.

\section{FRACCIÓN DE LLUVIA INTERCEPTADA POR EL FOLLAJE}

Se estima que en cada aguacero, el follaje, venciendo la gravedad y el viento, intercepta cerca de $1,3 \mathrm{~mm}$. Sin embargo, el follaje intercepta generalmente el $12 \%$ de la lluvia anual (Butler,1957). En otras palabras, se puede asumir que el mismo porcentaje de la lluvia mensual, es interceptado sin llegar al suelo. Linsley et al. (1958) mencionan que la lluvia anual interceptada en un bosque bien formado varía entre $10 \%$ y $20 \%$. En nuestro caso, asumiremos una retención del $12 \%$ para todos los casos.

Del análisis de lluvias de la estación meteorológica de San Josecito de Heredia, en el año de 1995, se observó que en cualquier mes que tuviese datos de lluvia, al menos habían caído 4 aguaceros pequeños. Esto implica, que al menos 5,2 $\mathrm{mm}$ al mes son interceptados por el follaje. Por lo anterior, precipitaciones mensuales menores de $5 \mathrm{~mm}$, se consideran que no infiltran ni escurren.

No se analiza la evaporación de las gotas de agua interceptadas por el follaje durante el aguacero, por considerarse que la atmósfera, al estar lloviendo, se encuentra con una humedad relativa saturada o casi saturada, lo que limita la evaporación, considerándola despreciable. Por lo tanto el coeficiente $(1-\mathrm{Ki})=(1-0,12)=0,88$. Por lo que la infiltración se representará de la siguiente forma:

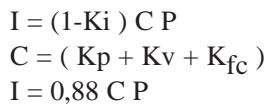




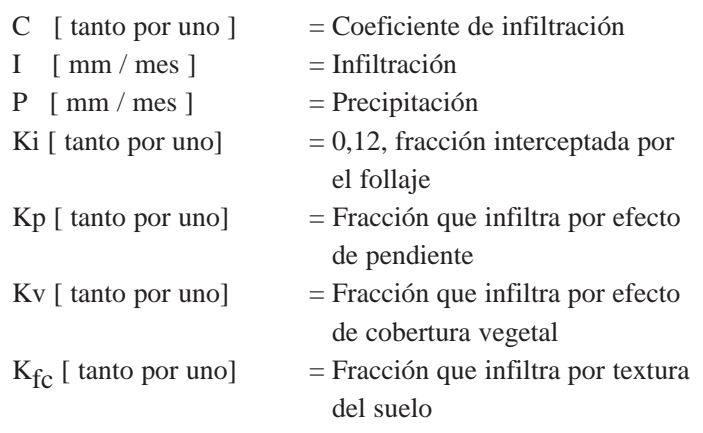

\section{INFILTRACIÓN POR TEXTURA DE SUELO}

En el coeficiente de infiltración, ecuación (1), se le ha asignado a la fracción que infiltra por textura de suelo valores entre 0,10 a 0,40. Estos valores dependen de los valores de infiltración básica (fc). Analizando bandas pluviométricas, y comparándolos con los valores de fc, se puede separar la fracción de lluvia que infiltra y la fracción de lluvia que escurre.

Con el propósito de separar la fracción de lluvia que infiltra, se realizaron varios estudios de bandas pluviográficas, en períodos de 10 minutos. El primero de ellos, en la zona del Valle del Río Tempisque (Elizondo, 1982), utilizando los datos de la estación meteorológica Hacienda Tempisque donde se analizaron aproximadamente seis años de lluvia. Posteriormente, se realizaron estudios similares, entre ellos: La zona de San Antonio de Belén (Gómez, 1987), que utiliza los datos de la estación meteorológica Aeropuerto Juan Santamaría, donde se analizó un año, formado por meses representativos de lluvia (tomando cada mes de diferentes años). La zona de Barranca (Arredondo, 1995), se estudió utilizando la estación meteorológica Puntarenas, en donde se analizó el año 1992. La zona de Zarcero (Montero \& Solano, 1986), con los datos de la estación meteorológica Palmira, donde se estudió un año formado con lluvias de meses representativos.

De las bandas pluviográficas de cada aguacero, medidas cada 10 minutos, se obtuvieron los valores de lluvia con intensidades menores o iguales al valor de infiltración básica del suelo (fc). Estos valores menores a fc, se sumaron hasta acumular los milímetros ocurridos en los aguaceros de un mes, para obtener lo que se designará como infiltración potencial (IP en mm/mes). Considerando estos valores, se preocedió a correlacionar los valores de precipitación mensual con los valores de infiltración potencial para cada valor de infiltración básica (fc en mm/día).

Esta infiltración potencial no considera la interceptación de lluvia en el follaje de las plantas. Además, asume que la infiltración se da únicamente durante el período de tiempo en que ocurrió el aguacero, considerando solo la capacidad de infiltración básica del suelo, sin tomar en cuenta el agua que infiltra durante el escurrimiento, una vez terminado el aguacero, por efecto de pendiente y cobertura vegetal. En otras palabras, la infiltración potencial no incluye los factores $\mathrm{Ki}, \mathrm{Kp}, \mathrm{Kv}$ indicados en la ecuación (1) y (2), incluyendo únicamente el factor $\mathrm{K}_{\mathrm{fc}}$, quedando de la forma $\mathrm{I}_{\mathrm{P}}=\mathrm{K}_{\mathrm{fc}} \mathrm{P}$.

Con base en los datos de los estudios realizados, se observa que los autores consideraron que las lluvias menores de $5 \mathrm{~mm}$ por mes, se infiltran totalmente. Por este motivo, lluvias menores de $5 \mathrm{~mm}$ mensuales no son consideradas en el análisis de correlación efectuado en este trabajo, por estimarse que son interceptadas por el follaje.

\section{Análisis de los datos de bandas pluviográfica}

De las bandas pluviográficas de cada aguacero, leídas con intervalos de diez minutos, se obtuvieron los valores de lluvia con intensidades menores al valor de infiltración básica del suelo ( $\mathrm{fc}$ ). Estos valores menores a fc, se sumaron hasta acumular los mm ocurridos en los aguaceros de un mes, para obtener la infiltración potencial (en $\mathrm{mm} / \mathrm{mes}$ ). Estos valores sumados ( $\mathrm{mm} / \mathrm{mes})$, se correlacionaron con los valores de precipitación mensual para cada valor de infiltración básica (fc en mm/día), como lo muestran las figuras 1,2 y 3.

Las tres figuras muestran correlaciones prácticamente lineales para cada valor de fc. La pendiente $\left(\mathrm{K}_{\mathrm{fc}}\right)$ de cada una de las rectas de mejor ajuste varía de acuerdo a los valores de fc. Las líneas de mejor ajuste están dadas por la siguiente ecuación: 


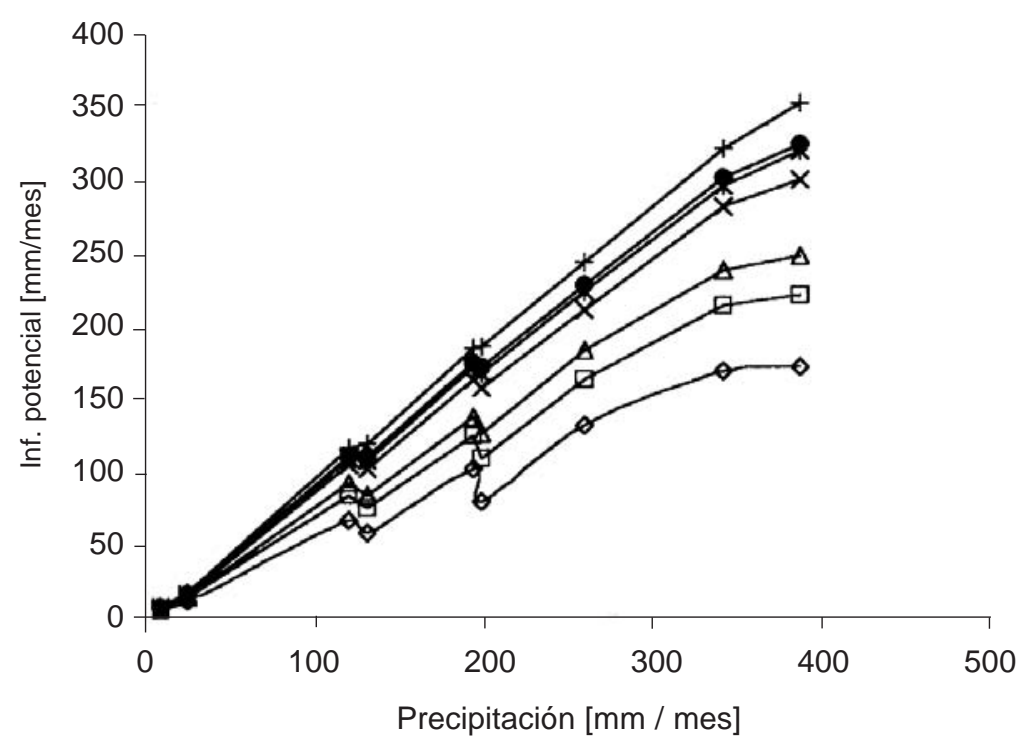

$$
\begin{aligned}
& \multimap \mathrm{fc}=84 \mathrm{~mm} / \mathrm{d} \\
& \square-\mathrm{fc}=161 \mathrm{~mm} / \mathrm{d} \\
& \triangle \mathrm{fc}=221 \mathrm{~mm} / \mathrm{d} \\
& \rightarrow-\mathrm{fc}=391 \mathrm{~mm} / \mathrm{d} \\
& \rightarrow-\mathrm{fc}=478 \mathrm{~mm} / \mathrm{d} \\
& -\mathrm{fc}=523 \mathrm{~mm} / \mathrm{d} \\
& +\mathrm{fc}=715 \mathrm{~mm} / \mathrm{d}
\end{aligned}
$$

Fig. 1: Correlación de Infiltración potencial durante el aguacero vrs precipitación para diferentes valores de fc. Datos de lluvia obtenidos de la estación meteorológica Hacienda Tempisque.

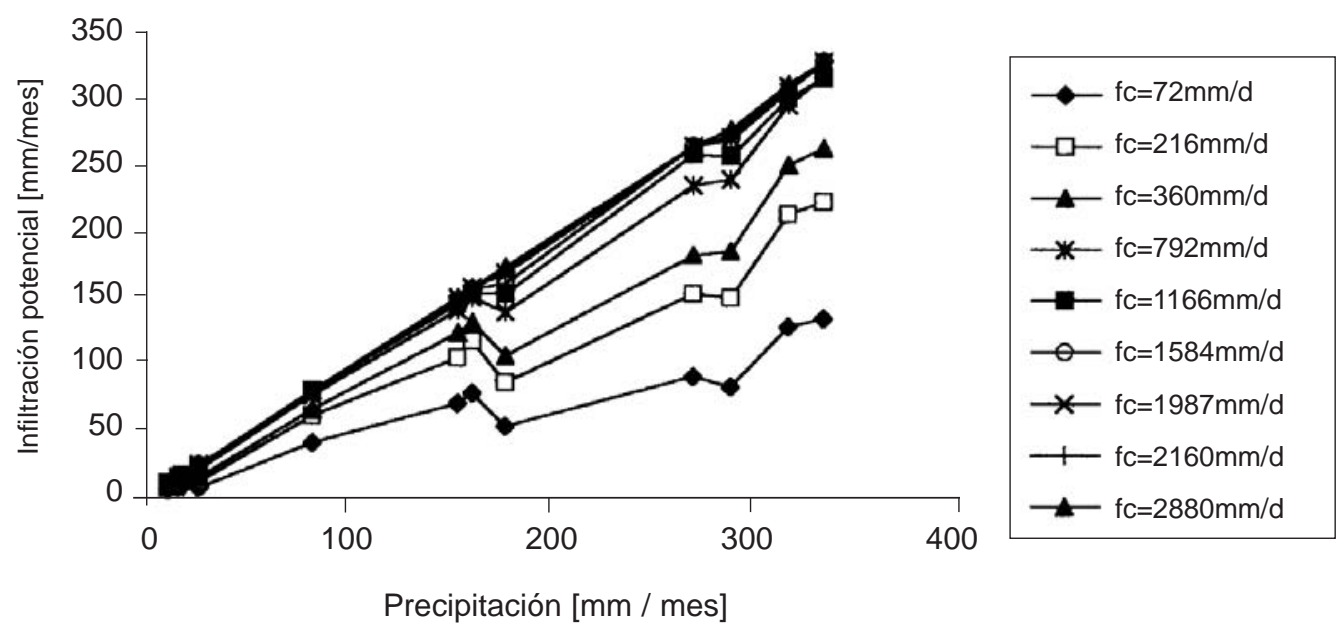

Fig. 2: Correlación de Infiltración potencial durante el aguacero vrs precipitación para diferentes valores de fc. Datos de lluvia obtenidos de la estación meteorológica Aeropuerto Juan Santamaría.

$$
\mathrm{IP}=\mathrm{P} . \mathrm{K}_{\mathrm{fc}}
$$

$$
\begin{array}{ll}
\mathrm{IP}[\mathrm{mm} / \mathrm{mes}] & =\text { Infiltración potencial } \\
\mathrm{P}[\mathrm{mm} / \mathrm{mes}] & =\text { Precipitación mensual mayor de } 5 \mathrm{~mm} \\
\mathrm{~K}_{\mathrm{fc}} & \text { Pendiente de la línea de mejor ajuste } \\
& \text { para cada valor de fc en las figuras } 1, \\
& 2 \text { y } 3 .
\end{array}
$$

Se obtuvieron los valores de la pendiente $\mathrm{K}_{\mathrm{fc}}$ de cada una de las líneas de mejor ajuste de las figuras 1,2 y 3 , los que se correlacionaron con los valores de infiltración fc. La línea de mejor ajuste obtenida de esta correlación es logarítmica y la ecuación obtenida para calcular $\mathrm{K}_{\mathrm{fc}}$ está indicada en las figuras 4,5 y 6. 


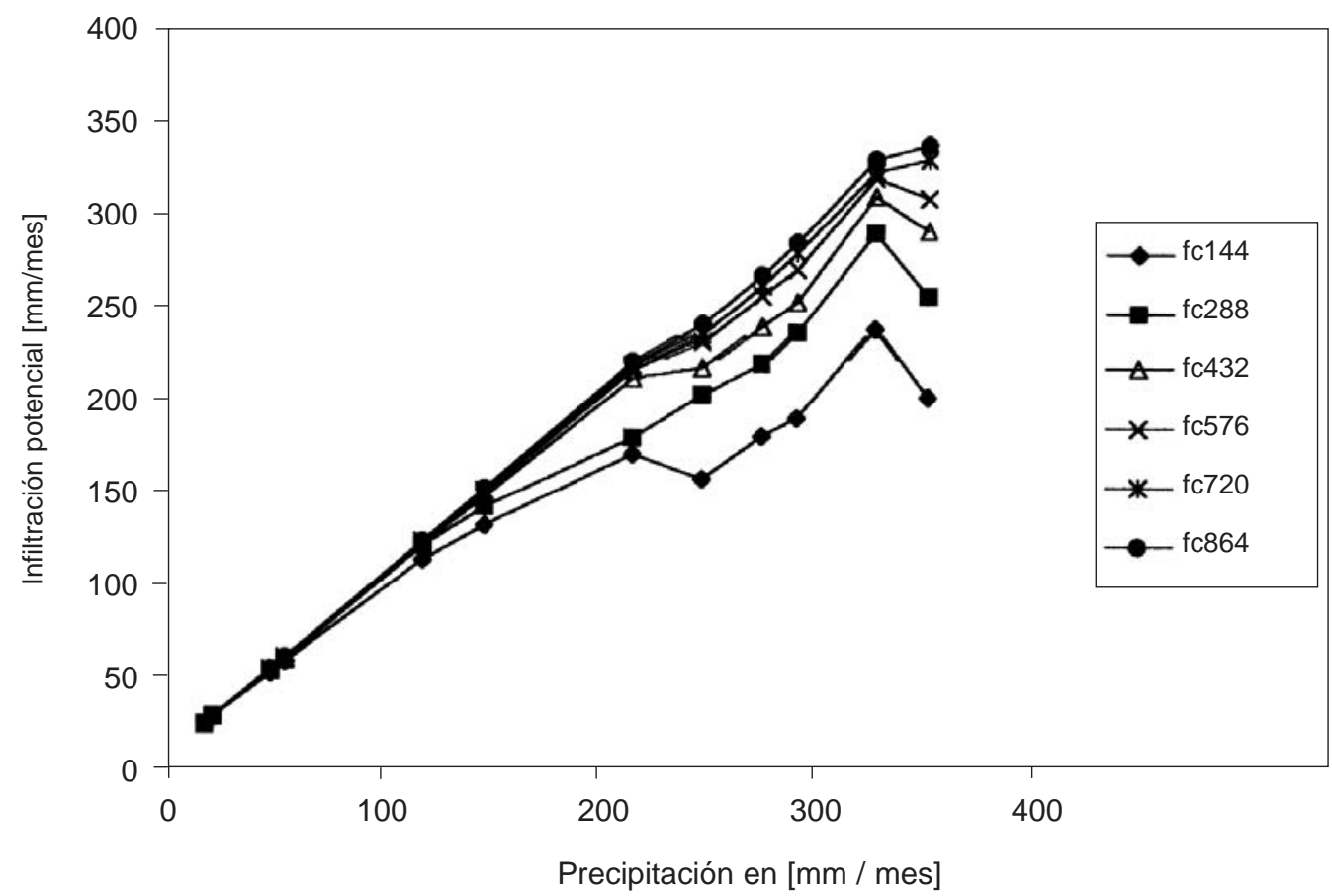

Fig. 3: Correlación de Infiltración potencial durante el aguacero vrs precipitación para diferentes valores de fc. Datos de lluvia obtenidos de la estación meteorológica Palmira..

\section{Hacienda Tempisque}

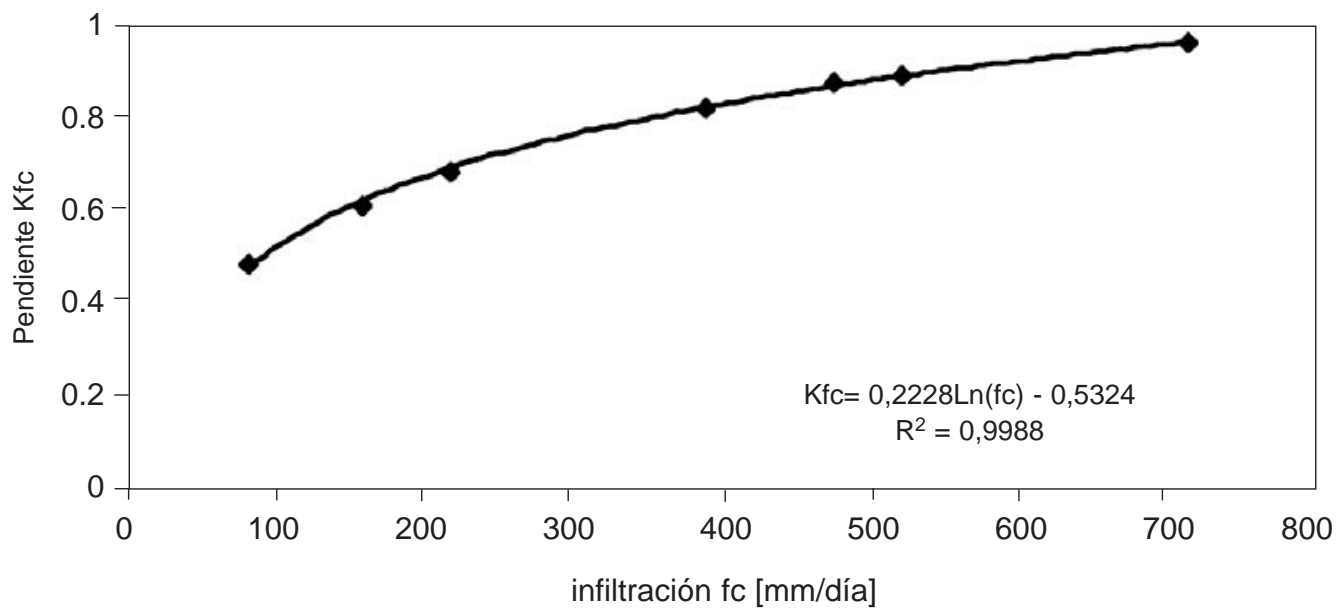

Fig. 4: Correlación de los valores de la pendiente Kfc (coeficiente de infiltración por textura de suelo) para diferentes valores de infiltración básica fc. Además se incluye la ecuación y el coeficiente de correlación $\mathrm{R}^{2}$. 


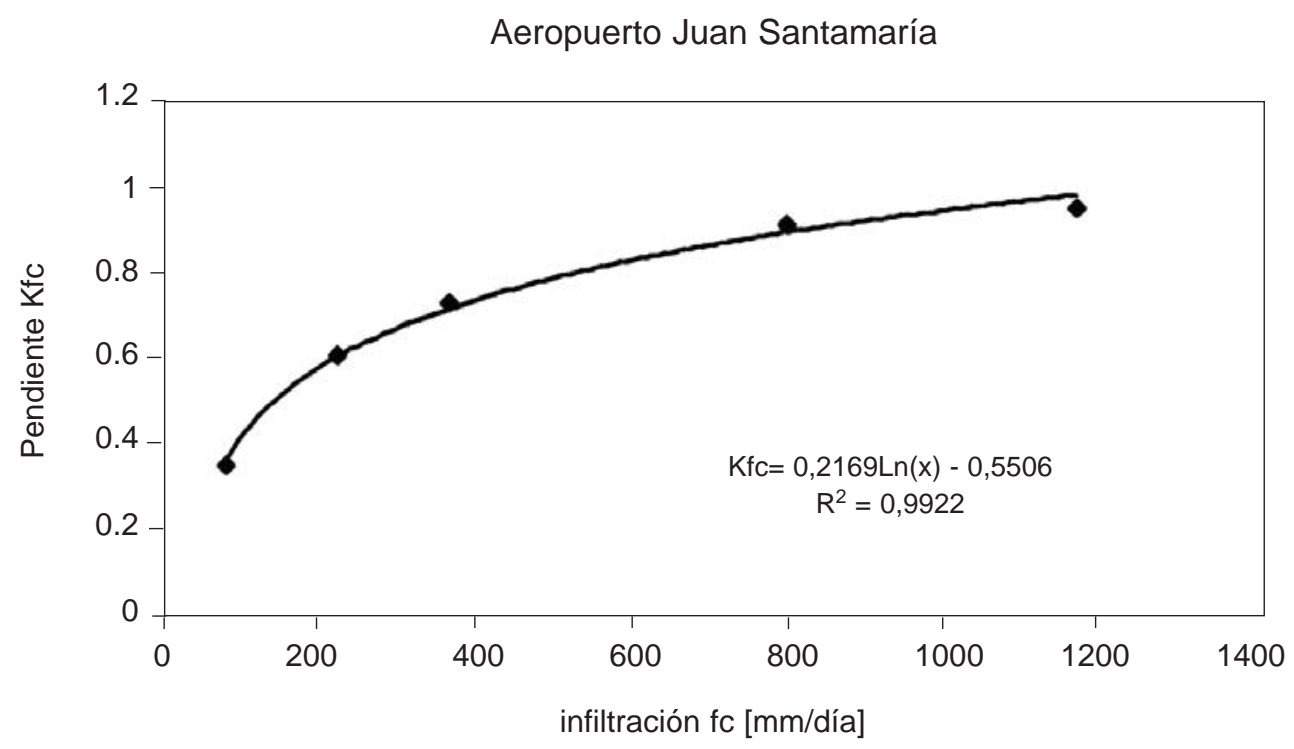

Fig. 5: Correlación de los valores de la pendiente $\mathrm{K}_{\mathrm{fc}}$ (coeficiente de infiltración por textura de suelo) para diferentes valores de infiltración básica fc. Además se incluye la ecuación y el coeficiente de correlación $\mathrm{R}^{2}$.

\section{Palmira}

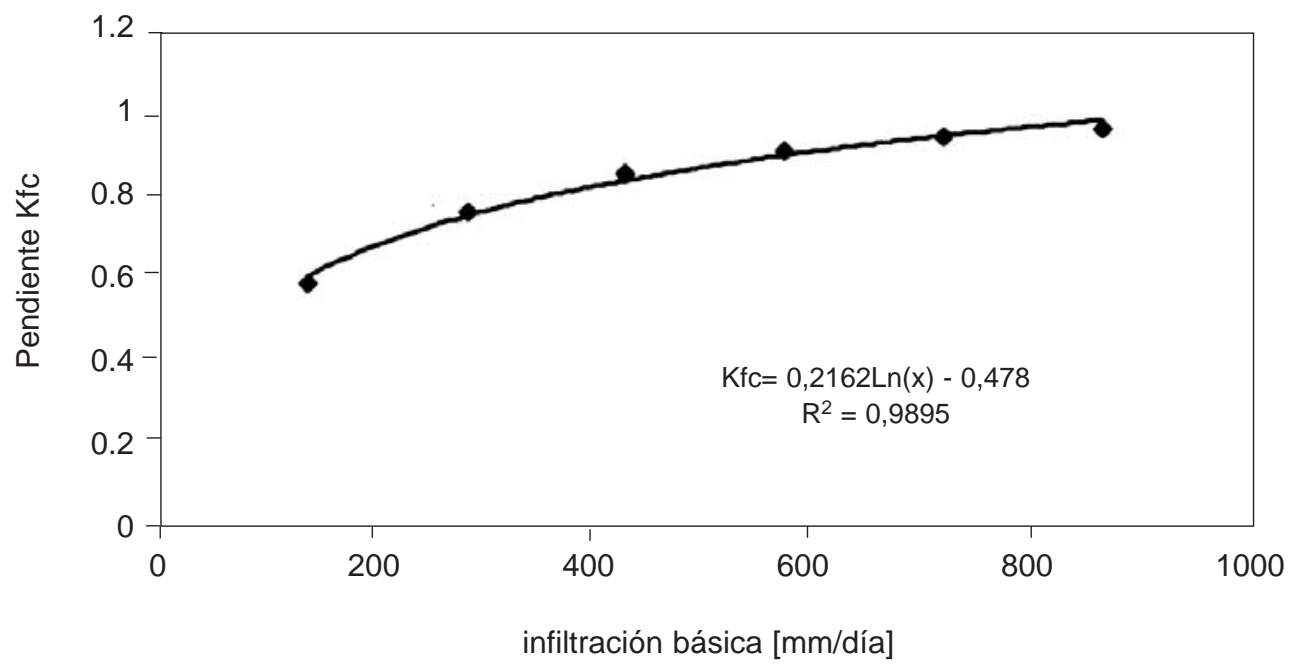

Fig. 6: Correlación de los valores de la pendiente $\mathrm{K}_{\mathrm{fc}}$ (coeficiente de infiltración por textura de suelo) para diferentes valores de infiltración básica fc. Además se incluye la ecuación y el coeficiente de correlación $\mathrm{R}^{2}$. 
Sustituyendo el valor de $\mathrm{K}_{\mathrm{fc}}$ en la ecuación (3), se tiene que la infiltración potencial esta dada por:

Zona del Valle del Tempisque, estación pluviográfica Hda Tempisque:

Ip $=\mathrm{P}\{0,22 \operatorname{Ln}(\mathrm{fc})-0,53\} \quad$ fc dentro del rango

20 - 1000 mm / día

Zona de San Antonio de Belén, estación pluviográfica Aeropuerto Juan Santamaría:

Ip $=\mathrm{P}\{0,22 \mathrm{Ln}(\mathrm{fc})-0,55\} \quad$ fc dentro del rango de $15-1200 \mathrm{~mm} /$ día

Zona de Zarcero, estación pluviográfica Palmira:

Ip $=\mathrm{P}\{0,22 \operatorname{Ln}(\mathrm{fc})-0,48\} \quad$ fc dentro del rango de $10-800 \mathrm{~mm} /$ día

Ip $[\mathrm{mm} / \mathrm{mes}] \quad=$ Infiltración potencial; no follaje,pendiente y cobertura

$\mathrm{fc}[\mathrm{mm} / \mathrm{día}]=$ Infiltración básica dentro del rango indicado

$\mathrm{P}[\mathrm{mm} / \mathrm{mes}]=$ Lluvia mayor de $5 \mathrm{~mm} / \mathrm{mes}$

\section{Análisis de datos de bandas pluviográfica de la estación Puntarenas}

El análisis de bandas fue realizado en un trabajo de tesis (Arredondo, 1995) para la zona de Barranca, utilizando los datos de la estación meteorológica de Puntarenas. En este caso se analizó el año 1992 con lecturas de bandas en períodos de diez minutos. Lamentablemente los resultados obtenidos de dicho análisis presentan serias dudas en su confiabilidad, por salirse completamente del patrón que presentan los casos anteriores, tales como Hacienda Tempisque, Aeropuerto Juan Santa María y Palmira. Esta anomalía se atribuye a agentes externos, tal como desperfecto en el pluviógrafo, bandas desperfectas u otros motivos. Por esta razón, estos datos no se incluyeron en los siguientes análisis.

\section{Ecuación General}

Para el estudio del coeficiente de infiltración a nivel nacional, se tomaron todas las bandas analizadas, separando para cada fc la fracción correspondiente a la infiltración y obtener una ecuación de infiltración potencial ( Ip ). Posteriormente se obtuvo la distribución del error de la ecuación con respecto a los valores de la infiltración obtenidos de las bandas.

En esta ecuación no se consideraron valores de precipitación menores de $5 \mathrm{~mm}$ mensuales, ni los datos de bandas de la estación pluviográfica de Puntarenas, por razones explicadas anteriormente.

\section{Infiltración potencial (Ip)}

De las bandas pluviográficas de cada aguacero, se obtuvieron los valores de lluvia con intensidades menores al valor de infiltración básica del suelo (fc). Estos valores menores a fc, se sumaron hasta acumular los mm ocurridos en los aguaceros de un mes, para obtener la infiltración potencial (IP en $\mathrm{mm} / \mathrm{mes}$ ). Se correlacionaron los valores de precipitación mensual con los valores de infiltración potencial para cada valor de infiltración básica (fc en $\mathrm{mm} /$ día), lo que se muestra en las figuras 1, 2 y 3 . Estas correlaciones son prácticamente lineales, donde la línea de mejor ajuste tiene una pendiente Kfc para cada valor de fc. Este valor de la pendiente representa el coeficiente de infiltración para una textura de suelo dada. Se graficaron los valores de Kfc para cada valor de fc, derivándose una ecuación de mejor ajuste (Fig. 7), la cual se puede aplicar como ecuación general en Costa Rica.

$\mathrm{K}_{\mathrm{fc}}=0,267 \ln (\mathrm{fc})-0,000154 \mathrm{fc}-0,723$

$\mathrm{K}_{\mathrm{fc}}$ [tanto por uno] = Coeficiente de infiltración por textura de suelo

fc [mm / día] = Infiltración básica del suelo

La ecuación general para determinar la infiltración potencial está dada por:

$\mathrm{Ip}=\mathrm{P}(0,267 \ln (\mathrm{fc})-0,000154 \mathrm{fc}-0,723)$ 


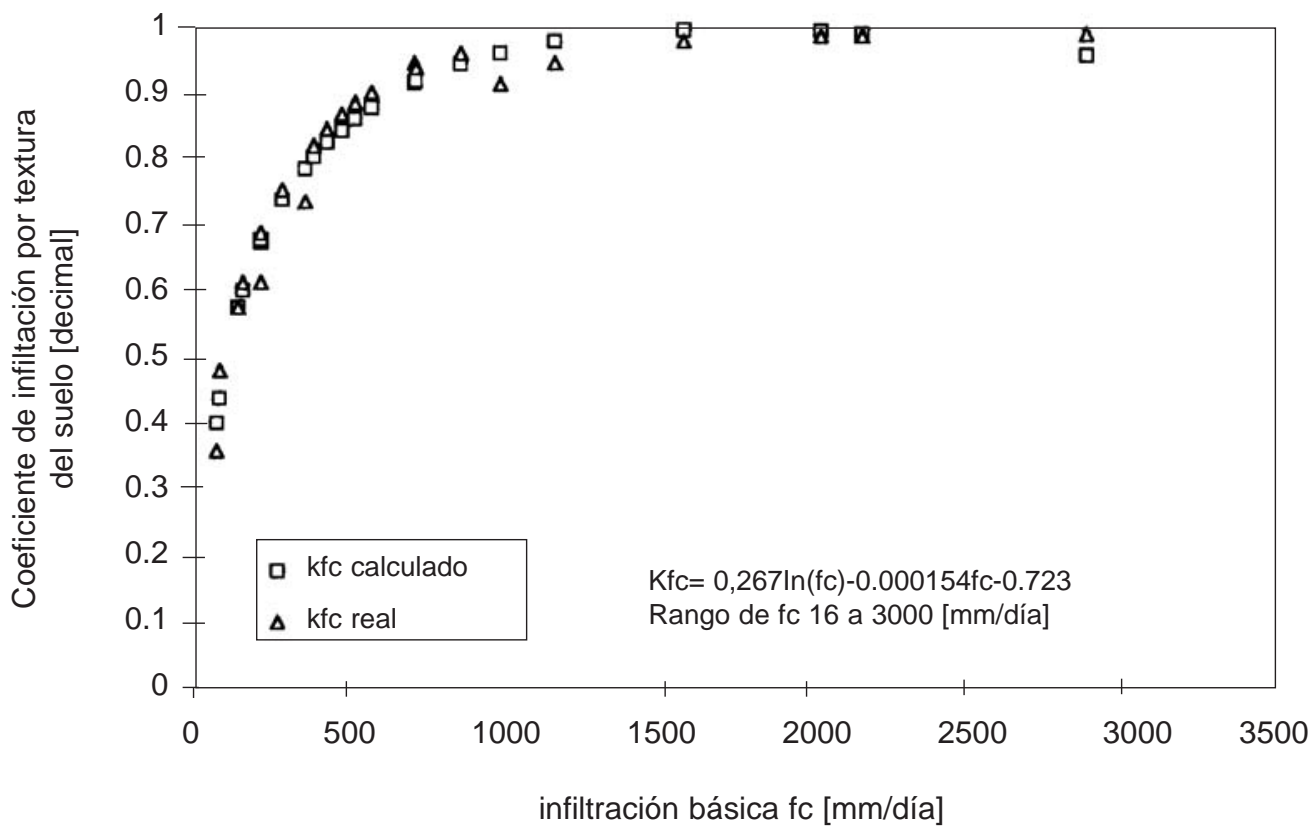

Fig. 7: Gráfica de $\mathrm{K}_{\mathrm{fc}}$ para calcular la fracción del coeficiente de infiltración debido a la textura del suelo, conociendo la infiltración básica del suelo. El rango a utilizar en fc es de 16 a 3000 mm/día. Se incluyen las estaciones Aeropuerto Juan Santamaría, Hacienda Tempisque y Palmira.

Ip $[\mathrm{mm} / \mathrm{mes}] \quad=$ Infiltración potencial general

$\mathrm{P}[\mathrm{mm} / \mathrm{mes}] \quad=$ Precipitación mensual mayores de $5 \mathrm{~mm} / \mathrm{mes}$.

fc $[\mathrm{mm} / \mathrm{día}] \quad=$ Infiltración básica del suelo, rango permisible 16 a 3000 [mm / día].

La correlación de la infiltración potencial calculada y la obtenida por bandas se muestra en la figura 8, en la cual se incluyeron las estaciones pluviométricas Aeropuerto Juan Santamaría, Hacienda Tempisque y Palmira. No se consideraron precipitaciones menores de $5 \mathrm{~mm} /$ mes.

Posteriormente, se realizó un análisis estadístico para determinar el error de la ecuación (5).

\section{Estimación del error de la ecuación general de infiltración potencial}

La distribución del error (Fig. 9) se asume con una distribución normal. Los datos con un porcentaje de error fuera del rango cubierto por la media, más-menos tres veces la desviación estándar (tres desviaciones estándar a la izquierda y tres a la derecha de la media, cubre el 99,73\% de la población), fueron eliminados, por considerarse que el error se debe a causas asignables a errores de medida u otros motivos. De esta forma se eliminan tres datos de valores extremos, de una población de 236 muestras (ITC, 1994).

El porcentaje de error de cada muestra se determinó, de la siguiente forma:

Porcentaje de error [tanto por uno] $=(\mathrm{A}-\mathrm{B}) / \mathrm{B}$

$$
\begin{aligned}
\mathrm{A}[\mathrm{mm} / \mathrm{mes}] & = \\
& \text { Infiltración potencial calculado } \\
& \text { con la ecuación }(5) . \\
= & \text { Infiltración potencial obtenido } \\
& \text { por medio de bandas. }
\end{aligned}
$$

Conocido el error de cada muestra, se agruparon los errores en rangos de 0,1, contándose el número de muestras dentro de cada rango, lo que equivale a la frecuencia. De esta forma se construyó la figura 9.

Los siguientes son los datos estadísticos de la figura 9: 


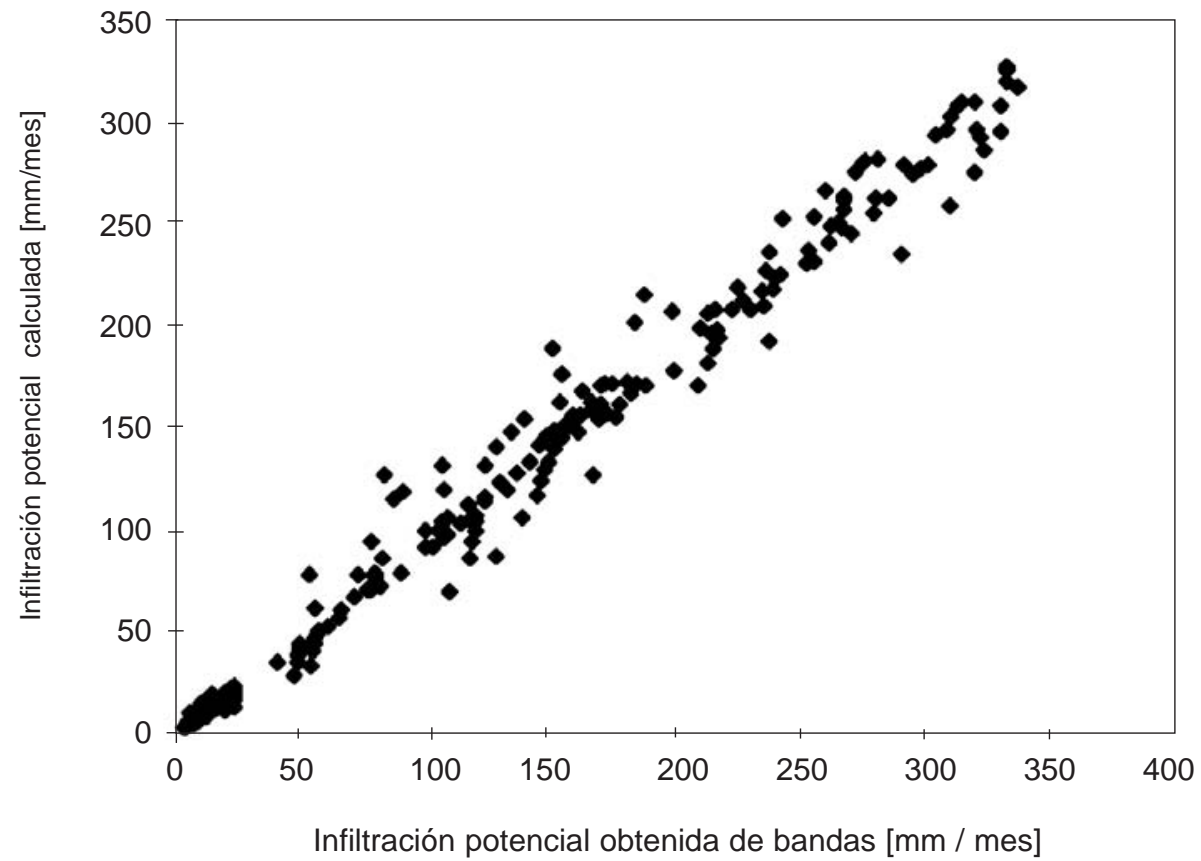

Fig. 8: Correlación de infiltración potencial obtenida por bandas vrs infiltración potencial calculada con ecuación (5). Las bandas analizadas incluyen las estaciones pluviográficas Aeropuerto Juan Santamaría, Hacienda Tempisque y Palmira.

Número de muestras

Desviación estándar del error

Promedio del error

Error con una confiabilidad del $68 \%$

Error con una confiabilidad del $95 \%$

$$
=233
$$

$=0,129$

$=-0,027$

$=12,9 \%$

$=25,8 \%$

De los datos estadísticos, podemos observar que en el $68 \%$ de los casos el error es menor al $12,9 \%$, lo que equivale al valor de una desviación estándar. En el $95 \%$ de los casos el error es menor al 25,8 \%, lo que equivale al valor de dos desviaciones estándar (ITC, 1994).



Fig. 9: Distribución del error de la infiltración potencial calculada con la ecuación (5). 


\section{CÁLCULO DE LA INFILTRACIÓN}

Para el cálculo de la infiltración se sugiere utilizar la ecuación (2), mencionada en el capítulo anterior de fracción de lluvia Interceptada por el follaje. En dicha ecuación se sustituye el parámetro $\mathrm{K}_{\mathrm{fc}}$ por el valor obtenido en la ecuación (4), quedando la ecuación general de la siguiente forma:

$\mathrm{I} \quad=0,88 \mathrm{C} \mathrm{P}$

$$
\begin{aligned}
& \mathrm{C}=\left(\mathrm{Kp}+\mathrm{Kv}+\mathrm{K}_{\mathrm{fc}}\right) \quad \text { para } \mathrm{Kp}+\mathrm{Kv}+\mathrm{K}_{\mathrm{fc}}<1 \\
& \mathrm{C}=1 \quad \text { para } \mathrm{Kp}+\mathrm{Kv}+\mathrm{K}_{\mathrm{fc}}>1 \\
& \mathrm{C}=0 \quad \text { para } \mathrm{P}<5 \mathrm{~mm} / \mathrm{mes} \\
& \mathrm{K}_{\mathrm{fc}}=0,267 \ln (\mathrm{fc})-0,000154 \mathrm{fc}-0,723 \\
& \mathrm{I}[\mathrm{mm} / \mathrm{mes}] \quad=\text { Infiltración mensual } \\
& \mathrm{P}[\mathrm{mm} / \mathrm{mes}] \quad=\text { Precipitación mensual } \\
& \mathrm{C} \text { [tanto por uno] = Coeficiente de infiltración igual } \\
& \text { o menor a uno } \\
& \mathrm{K}_{\mathrm{fc}} \text { [tanto por uno] }=\text { Fracción que infiltra por efecto } \\
& \text { de textura del suelo }
\end{aligned}
$$

Donde $\mathrm{Kv}$ y Kp son los coeficientes propuestos, en el capítulo de Coeficientes de infiltración, mostrados en el siguiente cuadro:

Por pendiente:

Muy plana

Plana

Algo plana

Promedio

Fuerte

Por cobertura vegetal

Cobertura con zacate menos $50 \%$

Terrenos cultivados

$0,02 \%-0,06 \%$

$0,3 \%-0,4 \%$

$1 \%-2 \%$

$2 \%-7 \%$

mayor de $7 \%$

Cobertura con pastizal

Bosques

Cobertura con zacate más de $75 \%$

\section{EJEMPLOS}

Los siguientes son ejemplos para obtener la infiltración con el procedimiento descrito, para la ecuación (6) :

Ejemplo 1- La precipitación del mes octubre es de $200 \mathrm{~mm}$. La infiltración básica del suelo es $85 \mathrm{~mm} /$ día. La zona se encuentra con cobertura de $50 \%$ zacate y $50 \%$ de bosque, con pendientes superiores al $7 \%$. La infiltración de octubre sería:

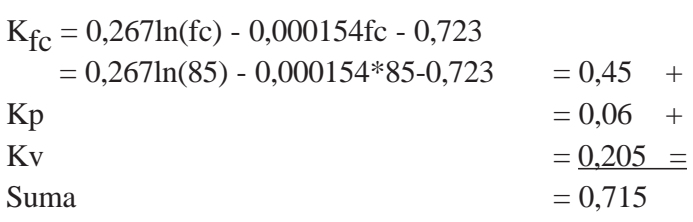

$\mathrm{C}=0,715$

El valor de $\mathrm{C}$ es menor de 1 y la precipitación es mayor de $5 \mathrm{~mm}$, por lo tanto se utiliza como coeficiente de infiltración el valor de 0,715 y la infiltración para el mes de octubre será:

$\mathrm{I}=0,88 \mathrm{C} \mathrm{P}=0,88^{*} 0,715^{*} 200=132 \mathrm{~mm}$

Ejemplo 2- La precipitación del mes mayo, es de $100 \mathrm{~mm}$. La infiltración básica del suelo es $200 \mathrm{~mm} /$ día. La zona se encuentra con cobertura de $80 \%$ zacate, con pendientes menores del $1 \%$. La infiltración de mayo sería :

$$
\begin{aligned}
& \mathrm{K}_{\mathrm{fc}}=0,267 \ln (\mathrm{fc})-0,000154 \mathrm{fc}-0,723 \\
& =0,267 \ln (200)-0,000154 * 200-0,723=0,66 \\
& \mathrm{Kp} \quad=0,20 \\
& \mathrm{Kv} \quad=\underline{0,21} \\
& \text { Suma } \quad=1,07
\end{aligned}
$$$$
\mathrm{C}=1
$$

0,06
La suma es mayor de 1 y la precipitación es mayor de $5 \mathrm{~mm}$, por lo tanto se utiliza como coeficiente de infiltración el valor de 1 y la infiltración para el mes de mayo será:

$\mathrm{I}=0,88 \mathrm{P}\left(\mathrm{Kp}+\mathrm{Kv}+\mathrm{K}_{\mathrm{fc}}\right)=0,88 * 100 * 1=88 \mathrm{~mm}$ 
Ejemplo 3- La precipitación del mes febrero es de $4 \mathrm{~mm}$. La infiltración básica del suelo es $200 \mathrm{~mm} /$ día. La zona se encuentra con cobertura de $80 \%$ zacate, con pendientes menores del $1 \%$. La infiltración para el mes de febrero sería:

$\mathrm{P}<5 \mathrm{~mm} / \mathrm{mes}$ implica $\mathrm{C}=0$

Al ser la precipitación de febrero menor de $5 \mathrm{~mm}$, la precipitación es interceptada por el follaje, por lo tanto no existe infiltración en el mes de febrero.

\section{CONCLUCIONES}

Este método simplifica enormemente el procedimiento para el cálculo de la precipitación que infiltra, necesaria para determinar la recarga a los acuíferos, utilizando la infiltración básica de los suelos y a la precipitación mensual en la zona, que de otra forma, implicaría realizar análisis diarios de las bandas pluviográficas.

Dependiendo de la distribución de lluvia, densidad y tipo de la vegetación, el porcentaje anual de la lluvia interceptada por el follaje (Ki) puede variar entre $10 \%$ y $20 \%$ en un bosque (Linsley et al., 1958). Sin embargo Butler (1857) menciona que este porcentaje generalmente corresponde al $12 \%$. Debido a la complejidad para determinar la retención de lluvia, se ha tomado el $12 \%$, como un valor de retención mensual para todos los casos, lo que asume que el $88 \%$ de la lluvia mensual es la que llega al suelo. Futuras investigaciones, en que se incluya el uso del suelo y vegetación de Costa Rica, podrían variar un poco dicho porcentaje.

La infiltración potencial (Ip), contiene únicamente la cantidad de lluvia que infiltra por efecto de la textura del suelo, $\mathrm{Ip}=\mathrm{P}^{*} \mathrm{~K}_{\mathrm{fc}}$ ecuación (3). Esta cantidad es la que se infiltra mensualmente durante el tiempo que dura cada uno de los aguaceros ocurridos en el mes, no considera la lluvia interceptada por el follaje, ni la infiltración que se produce durante el escurrimiento superficial después de terminado el aguacero. La infiltración, que se produce mientras escurre el agua sin que esté lloviendo, es evaluada median- te los coeficientes $\mathrm{Kp}+\mathrm{Kv}$ (fracción que infiltra por efecto de pendiente + fracción que infiltra por efecto de covertura vegetal), que son los que indican la aceleración o retraso del escurrimiento superficial, permitiendo una mayor o menor infiltración del agua que escurre.

En estudios realizados en zonas volcánicas altas cerca de la Laguna de Apoyo (Granada Nicaragua), se ha observado que por efecto de textura de suelo, el agua de lluvia que llega a la superficie del mismo, infiltra el $100 \%$. Concluyendo, que existen zonas de alta infiltración básica, en que el coeficiente de infiltración (C) llega a un valor igual a 1 , debido únicamente, al efecto de textura de suelo.

Para determinar el porcentaje de error de la infiltración potencial (Ip) estimada con la ecuación (5), se compararon 236 muestras de (Ip), analizadas por diferentes autores, mediante lecturas de bandas pluviométricas. En 160 casos (68\% de las muestras), el error fue menor de 13\% en cualquiera de ellas y en 224 casos (95\% de las muestras) el error es menor de $26 \%$, en cualquiera de ellas. El error promedio es de $-0,027 \mathrm{~mm}$. Se concluye, que promediando la infiltración de 12 meses, el error anual será muy bajo, casi despreciable. Sin embargo, puede darse el caso que en 95 meses analizados; ninguno de ellos, tendrá un error mayor del $26 \%$. Al realizarse, generalmente los balances de infiltración como un promedio de varios meses, la ecuación (5), es bastante aceptable.

\section{REFERENCIAS}

ARREDONDO L., S., 1995: Evaluación de la vulnerabilidad a la contaminación de los acuíferos, Barranca y el Roble, Puntarenas, Costa Rica. - 76 págs. Escuela Centroamericana de Geología, Universidad de Costa Rica [Tesis Lic.].

BUTLER, S., 1957: Engineering Hydrolgy. 356 págs. Prentice Hall Inc. USA.

CHOW, V.T., MAIDMENT, D.R. \& MAYS, L.W., 1994: Hidrología aplicada. - 584 págs. Mac Graw Hill, Santa Fé Bogotá. 
ELIZONDO M., J., 1982: Estudio hidrogeológico con fines de riego en la margen derecha del río Tempisque. - 39 págs. SENAS [Inf. técnico $\mathrm{N}^{\mathrm{o}} 152$ ).

GOMEZ C., A., 1987: Evaluación del potencial de los acuíferos y diseño de las captaciones de agua subterránea en la zona de puente mulas, provincia de Heredia Costa Rica. - 66 págs. Escuela Centroamericana de Geología, Universidad de Costa Rica [Tesis Lic.].

ITC, 1994: Frecuency analysis. - 74 págs. ITC, Holanda.
LINSLEY, R., KOHLER, M. \& PAULUS, L., 1958: Hidrology for Engienners. - 340 págs. Mc Graw Hill, New York.

MURILLO, M.W. \& SOLANO, B. E., 1986: Análisis y utilización de los recursos subterráneos en Zarcero. - 86 págs. Escuela Ingeniería Civil, Universidad de Costa Rica [Tesis Lic.].

ONU, 1972: Manual de instrucciones, estudios hidrológicos. - 540 págs. ONU Publ.N ${ }^{\circ}$ 70.; San José, Costa Rica. 